Extreme Adaptive Optics Testbed: Results and Future Work

J. W. Evans, G. Sommargren, L. Poyneer, B. Macintosh, S. Severson, D. Dillon, A. Sheinis, D. Palmer, J. Kasdin, S. Olivier

July 21,2004

Astronomical Instrumentation for SPIE Glasgow, United Kingdom June 21, 2004 through June 25, 2004 
This document was prepared as an account of work sponsored by an agency of the United States Government. Neither the United States Government nor the University of California nor any of their employees, makes any warranty, express or implied, or assumes any legal liability or responsibility for the accuracy, completeness, or usefulness of any information, apparatus, product, or process disclosed, or represents that its use would not infringe privately owned rights. Reference herein to any specific commercial product, process, or service by trade name, trademark, manufacturer, or otherwise, does not necessarily constitute or imply its endorsement, recommendation, or favoring by the United States Government or the University of California. The views and opinions of authors expressed herein do not necessarily state or reflect those of the United States Government or the University of California, and shall not be used for advertising or product endorsement purposes. 


\title{
Extreme Adaptive Optics Testbed: Results and Future Work
}

\author{
Julia Wilhelmsen Evans*a, b, Gary Sommargren ${ }^{\mathrm{a}}$, Lisa Poyneer ${ }^{\mathrm{a}}$, Bruce Macintosh ${ }^{\mathrm{a}, \mathrm{c}}$, Scott \\ Severson $^{\mathrm{c}}$, Daren Dillon ${ }^{\mathrm{c}}$, Andrew Sheinis ${ }^{\mathrm{c}}$, David Palmer ${ }^{\mathrm{a}, \mathrm{c}}$, Jeremy Kasdin ${ }^{\mathrm{d}}, \mathrm{S}^{\mathrm{a}}$ cot Olivier $^{\mathrm{a}}$ \\ ${ }^{a}$ Lawrence Livermore National Laboratory, ${ }^{b}$ University of California/Davis, ${ }^{\mathrm{C}}$ University of \\ California/Santa Cruz, ${ }^{\mathrm{d}}$ Princeton University
}

\begin{abstract}
"Extreme" adaptive optics systems are optimized for ultra-high-contrast applications, such as ground-based extrasolar planet detection. The Extreme Adaptive Optics Testbed at UC Santa Cruz is being used to investigate and develop technologies for high-contrast imaging, especially wavefront control. A simple optical design allows us to minimize wavefront error and maximize the experimentally achievable contrast before progressing to a more complex set-up. A phase shifting diffraction interferometer is used to measure wavefront errors with sub-nm precision and accuracy. We have demonstrated RMS wavefront errors of $<1.3 \mathrm{~nm}$ and a contrast of $>10^{-7}$ over a substantial region using a shaped pupil. Current work includes the installation and characterization of a 1024-actuator Micro-Electro-MechanicalSystems (MEMS) deformable mirror, manufactured by Boston Micro-Machines, which will be used for wavefront control. In our initial experiments we can flatten the deformable mirror to 1.8-nm RMS wavefront error within a control radius of 5-13 cycles per aperture. Ultimately this testbed will be used to test all aspects of the system architecture for an extrasolar planet-finding AO system.
\end{abstract}

Keywords: high-contrast imaging, extrasolar planet detection, interferometery, adaptive optics, micro-electricalmechanical-systems

\section{Introduction and Motivation}

The imaging of extrasolar planets, either from spacecraft or ground-based adaptive optics systems, promises to be one of the most interesting scientific developments of the next decade, and also one of the most challenging. A halo of scattered light from the star will limit contrast and our ability to image a planet. This halo contains components due to both diffraction and a speckle pattern due to residual phase errors ${ }^{1}$. High-contrast imaging requires that both components be controlled. Wavefront errors can come from both dynamic terms such as residual atmospheric phase, and quasi-static sources such as internal optical errors and errors in calibration of the adaptive optics system ${ }^{2}$. Atmospheric phase errors produce a PSF halo that becomes smoother with longer integrations, but scattered light caused by static wavefront errors will produce a residual pattern of speckles that will not smooth out over time ${ }^{1}$. Simulations indicate that an ExAO system designed to achieve $10^{-7}$ contrast will require between $1-2 \mathrm{~nm}$ of RMS static wavefront error over the range of spatial frequencies controlled by the deformable mirror ${ }^{3}$.

The extreme adaptive optics testbed is designed to test the limits of achievable contrast in the lab and to develop highcontrast technologies for a future extrasolar planet imager.

\section{Experimental setup}

\subsection{Testbed Layout}

Scattered light from diffraction and wavefront errors need to be controlled to operate the testbed in the high-contrast regime. The simple optical design was implemented with high-quality optics to minimize wavefront errors, while a

*wilhelmsen3@llnl.gov 


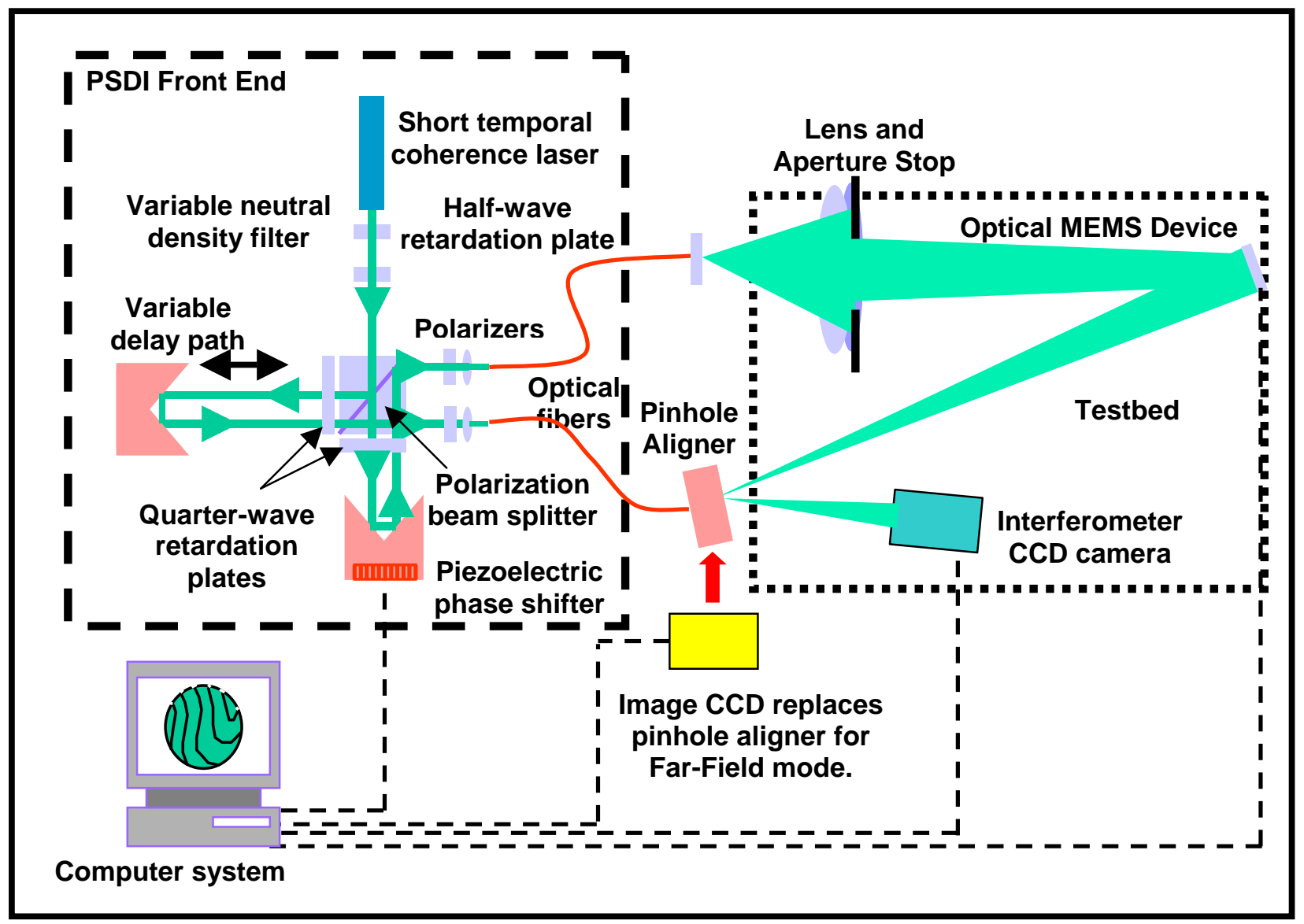

Figure 1: Layout of ExAO testbed including the phase shifting diffraction Interferometer. The testbed is shown in wavefront measurement mode. For far field mode the pinhole aligner is replaced by the image CCD.

phase shifting diffraction interferometer (PSDI) provides precision metrology of the system. Diffraction is suppressed over specific regions of the image plane using shaped pupils such as the prolate spheroid pupil ${ }^{4}$. The results presented used a pupil made for Princeton university ${ }^{5}$ using laser cutting. Shaped pupils are an excellent way to suppress diffraction in a testbed environment because they are relatively simple to use and do not introduce any phase errors into the system. For example, a Lyot Coronagraph would require additional optics for a reimaged pupil, increasing the complexity of the system and potentially introducing wavefront errors ${ }^{6}$. Shaped pupils do not produce a uniform region of suppression, like Lyot Coronagraphs, making them less suited to the final instrument, but this limitation is not a problem in a laboratory setting.

There are two modes of operation for the ExAO testbed: Wavefront measurement mode and Far Field mode. In Far Field mode only the measurement leg of the PSDI is used and the pinhole aligner is replaced with a CCD camera. In the initial experiments a flat mirror was used in place of the MEMS deformable mirror to measure the best contrast of the system. The MEMS mirror has been installed and we are attempting to reproduce the contrast results achieved with the flat mirror. The layout is shown in Figure 1.

\subsection{Phase Shifting Diffraction Interferometer}

The phase shifting diffraction interferometer uses the fundamental process of diffraction to generate near perfect spherical wavefronts ${ }^{7}$. Both reference and measurement beams are produced by single mode fibers, which interfere at the face of the pinhole aligner. A pinhole emits the reference beam and the polished front surface reflects the measurement beam. The resulting interference pattern is measured and numerical propagation calculates the wavefront 
of the system under test. Using a single mode fiber to produce the reference beam eliminates the need for a reference surface and allows the PSDI to make an absolute measurement of the wavefront. The fibers also filter out high-order errors that are introduced by the front-end optics. All other optics can be kept to a minimum. The PSDI can still utilize phase shifting and the standard algorithms for data analysis of conventional interferometry. The PSDI was developed for the extreme ultra-violet lithography project at Lawrence Livermore National Laboratory and can achieve sub-nm precision $^{8}$.

\section{Results}

Two types of data are currently taken on the ExAO testbed: wavefront measurements and PSF measurements. From the PSF measurements we can directly measure the achievable contrast of the system. The wavefront measurements indicate if the system is meeting our goals for wavefront quality and provide a consistency check for the PSF measurements.

\subsection{Wavefront Measurements}

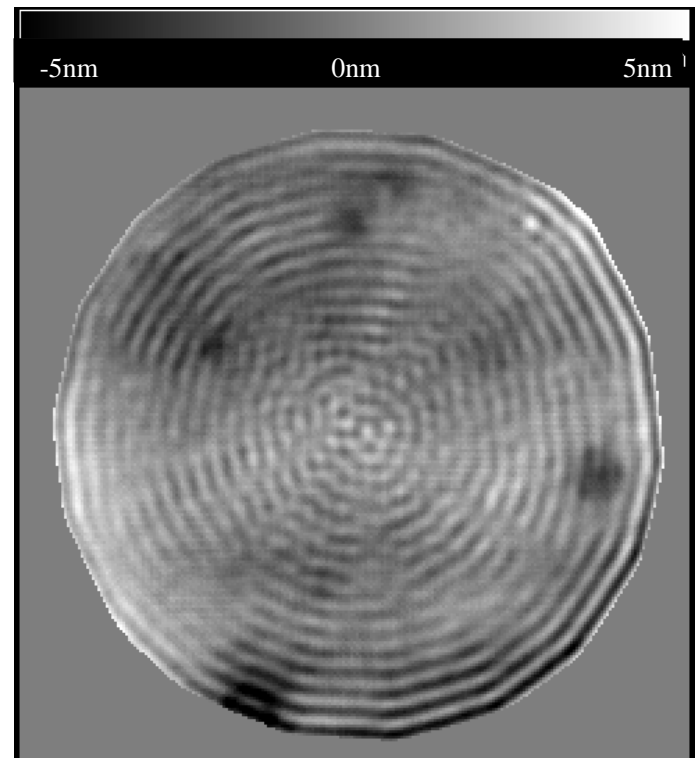

Figure 2: Sample wavefront measurement from ExAO testbed. The RMS wavefront error is $<1.3 \mathrm{~nm}$.
The ExAO testbed (with the flat mirror replacing the MEMS DM) has consistently been measured by the PSDI to have a RMS wavefront error of $<2 \mathrm{~nm}$ over a $10-\mathrm{mm}$ pupil. Tilt, piston and focus aberrations are removed in processing. The primary aberrations remaining are astigmatism and coma. The sample wavefront in Figure 2, for example, has total RMS wavefront error of $1.3 \mathrm{~nm}$ including $0.7 \mathrm{~nm}$ of astigmatism, and $0.8 \mathrm{~nm}$ of midfrequency aberration. The ringing artifacts visible in the image accounts for about $0.5 \mathrm{~nm}$ and is caused by truncation effects at the pinhole aligner. Simulations indicate that this level of wavefront error is easily sufficient to achieve contrast $>>10^{7}$.

\subsection{Contrast measurements}

There are several error sources in high-contrast imaging that must be reduced to achieve good results: insufficient dynamic range, scattered light from the optical system, and CCD saturation effects.

Ideally the contrast of an image in a given region could be measured by comparing the peak of the PSF to the average intensity of the PSF in that region in a single image. The CCD in the ExAO system has a dynamic range of

approximately four orders of magnitude. A contrast measurement of $10^{-7}$ can only be made as a composite of two or more images. Typically an unsaturated (Figure 3 ) and a saturated image that are separated by $4-5$ orders of magnitude are used. The intensity is reduced by four orders of magnitude using neutral density filters and a fifth order is obtained by increasing the integration time. The ND filters are inserted into the PSDI front-end right before the measurement beam is launched into the fiber. Small amounts of wedge in the filters will change coupling of light into the fiber, varying the attenuation from the filter. This effect is minimized by keeping the fiber launchers well aligned (they are temperature sensitive) and by putting the ND filters close to the launchers. For our work the effect was still significant and a power meter was used to characterize the attenuation caused by the ND for every experiment. The ND filters and laser power would be adjusted to return an

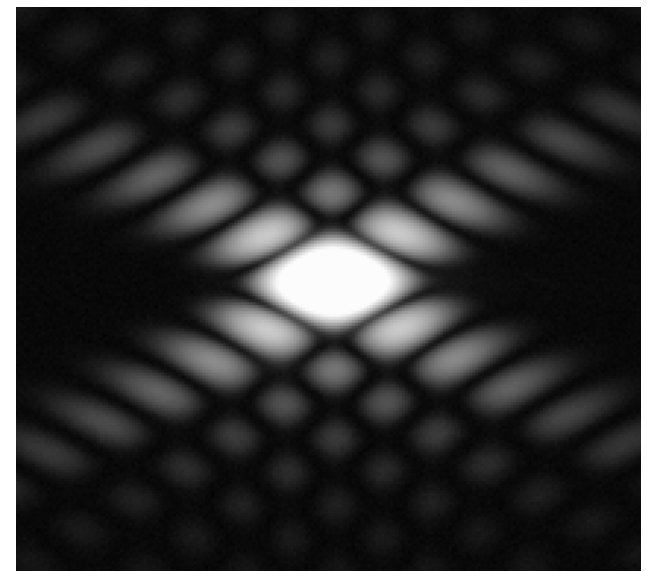

Figure 3: Unsaturated PSF of the prolate spheriod pupil provided by Princeton University displayed with a log scale. 
attenuation of $10^{4}$ for the unsaturated image. The filters were removed and the integration time increased from 0.01 to 0.1 seconds for the saturated image. We also ran tests to determine the power variability in short exposures (either variability in the laser power or in the shutter speed) and choose our integration times to fall within the region of low variability.

The testbed is housed in an opaque enclosure, but scattered light from elements in the optical path is a problem. The shaped pupils need to be anodized and the path off of the pupil and into the camera is baffled with dark beam tubes (diameter >>beam size). Baffling with dark fabric was necessary in some places to reduce reflected light off the smooth side of the opaque enclosure. We tested our control over scattered light by comparing dark frames where the laser was shuttered to dark frames where the laser was blocked at the flat. The enclosure and small beam size also reduce air turbulence or bench "seeing", which was not a problem in these experiments.

When the ND filters are removed and the integration time is increased the CCD is highly saturated. Saturation effects in the CCD cause photoelectrons from the core of the image to bleed into the null regions of the diffraction pattern, where you wish to measure intensity. The slow f/\# of our system allows us to move the far-field camera approximately $15 \mathrm{~mm}$ behind the focal plane without loss of contrast. A mirrored beam block is introduced at the focal plane to block the core, but not the wings of the PSF. The block is aligned to ensure that the light from the core does not scatter into the CCD or return along the beam path, and the reverse side is painted black. The horizontal position of the block is optimized by hand to minimize the amount of bleeding from the core, while still measuring the contrast at the small inner working angle (4-5 $\lambda / \mathrm{D})$ that the shaped pupils should achieve.

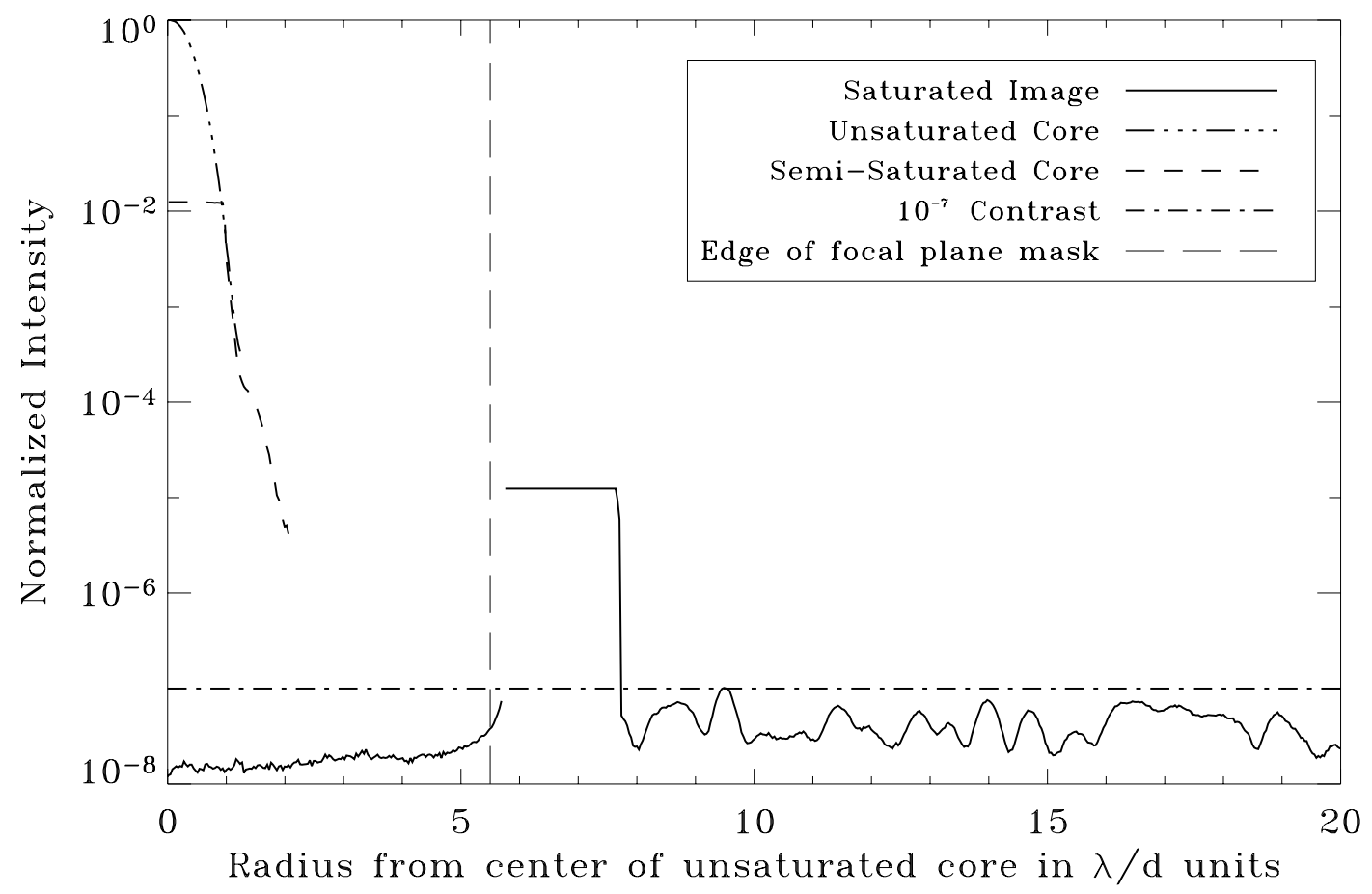

Figure 4: Slice through the PSF produced with a prolate spheroid pupil from Princeton University. Three data sets are included in this graph. The solid line is the saturated image. The vertical dotted line represents the edge of the mirrored focal plane block. To the right of the block the saturated image is affected by bleeding from nearby pixels. Contrast below $10^{-7}$ is achieved to the right of the graph. To the left of the block, the noise floor of the system at $10^{-8}$ can be seen. The two dotted lines trace out the core of the image.

For analysis each data set of 12 frames is averaged and the corresponding averaged dark frame is subtracted. Then each image is scaled according to the measured attenuation from the ND and integration time to be comparable with the saturated image. All of the images are normalized to the peak of the scaled unsaturated image. Additional images with attenuation in between the saturated and unsaturated images can be used to investigate the connection between the core 
and wings of the PSF.

Figure 4 is a composite of three data sets demonstrating a contrast of $>10^{-7}$. Bleeding from near by pixels causes the bright area in the lineout from the saturated image. The vertical dotted line indicates where the mirrored block was when the saturated image was recorded. Behind it the noise floor of the system is at the $10^{-8}$ level. The pupil used for this data was optimized for $10^{-10}$
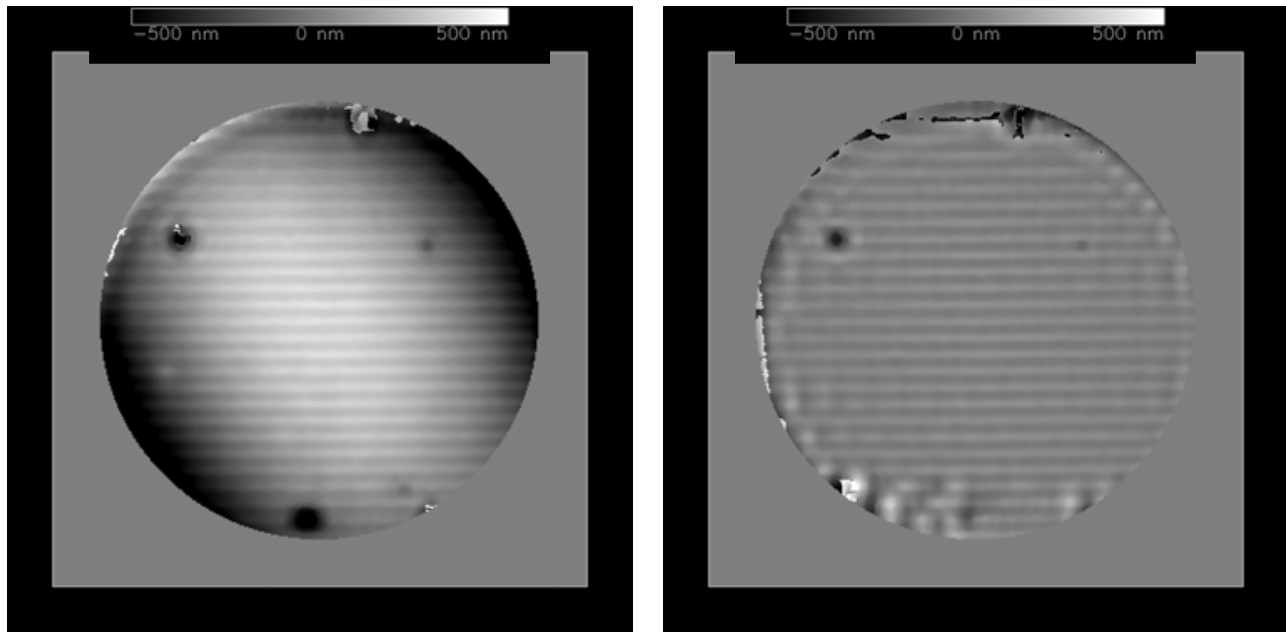

Figure 5: The uncorrected and corrected wavefronts from the initial MEMS DM tests with tilt removed in processing. The horizontal structure in the wavefronts is caused by the physical structure in the MEMS mirror. contrast, however it only achieves a contrast between $10^{-7}$ and $10^{-8}$. The noise floor is below the measured contrast indicating that the system is limited by the pupil not the noise floor of the system. Edge roughness in the pupil will cause light to scatter into the regions you wish to measure. Other manufacturing techniques may be investigated in the future, but the current set-up achieves the contrast necessary for the ExAO systems being specified.

\subsection{Initial MEMS results}

The MEMS is operated in a closed-loop mode using the PSDI to provide wavefront measurements. To operate the MEMS device alignment and calibration routines must be run. The alignment routine depresses four actuators on the device and aligns them with the corresponding phase measurement. The calibration routine currently uses the response of four actuators at nine voltages to generate a voltage calibration curve, which is fit linearly for our initial tests. Because the response of the MEMS actuators is non-uniform a more rigorous calibration would characterize the gain of each actuator. After alignment and calibration the closed loop routine is run. The PSDI software acquires an interferogram and

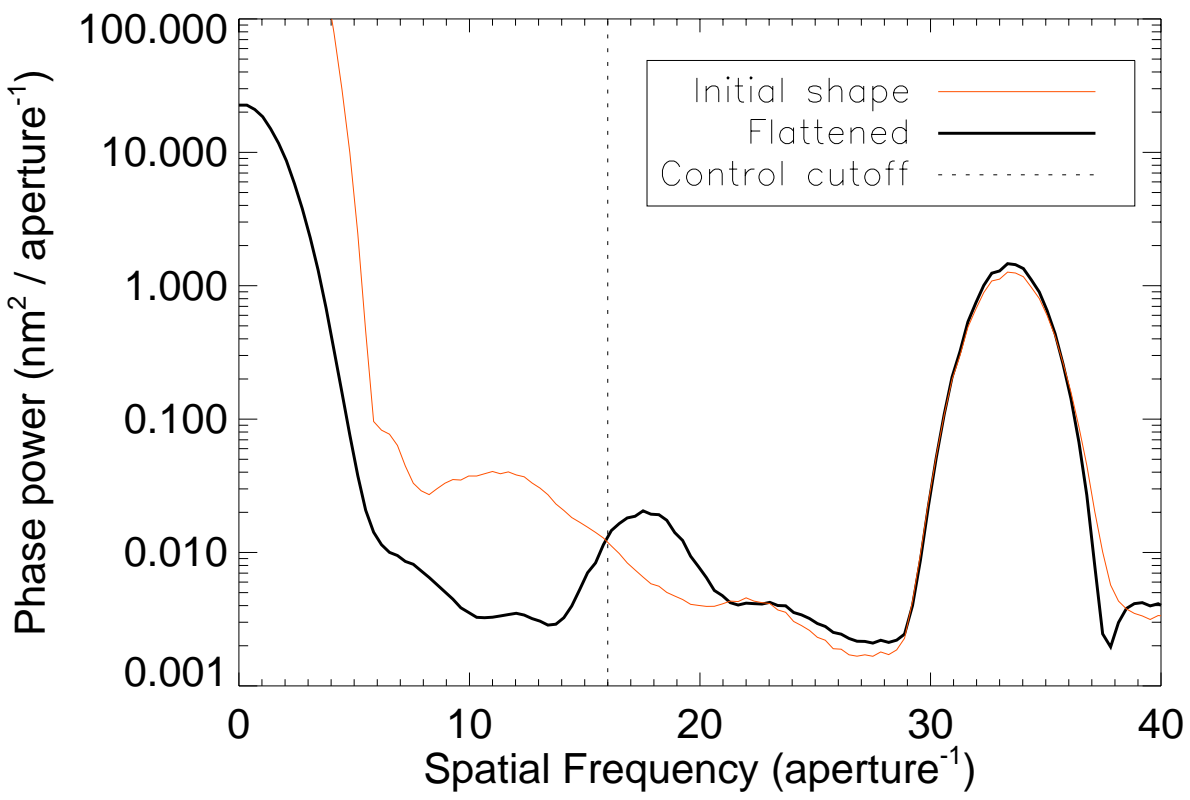
numerically back-

Figure 6: The power spectrum of the corrected and uncorrected wavefronts of the MEMS DM. The closed loop system converges to an RMS wavefront error of $1.8 \mathrm{~nm}$ in the control radius of 5 to 13 cycles per aperture. Tilt was not corrected by the initial control system. 
propagates it to the plane of the MEMS (approximately the pupil plane of the system). The IDL code converts the phase measurements into MEMS commands and applies the commands to the mirror. Better calibration will cause the closed loop to converge more quickly.

The closed loop system converges after 6 iterations to an RMS wavefront error of $1.8 \mathrm{~nm}$ in a control radius of 5 to 13 cycles per aperture (from $\sim 5 \mathrm{~nm}$ initially). The low order errors (primarily tilt) that remain on the device were not being fully corrected by the initial control system, but we have achieved the wavefront error needed for an ExAO system in a control radius. Figure 5 shows the corrected and uncorrected wavefronts of the MEMS DM with a circular aperture. The horizontal structure is caused by the physical structure of the MEMS. Figure 6 is the power spectrum of the two wavefronts.

\section{Conclusions and Future work}

The Extreme Adaptive Optics testbed has demonstrated that $10^{-7}$ contrast needed to image young Jupiter like planets is achievable in a laboratory setting. We are using this high-contrast environment to test subsystems for an extrasolar planet imager. MEMS deformable mirror technology is the first subsystem under test and we have demonstrated the necessary flattening over a specific control radius. Future work with the mems device will enable the testing and specification of other ExAO subsystems such as s a spatially filtered wavefront sensor ${ }^{9}$.

\section{ACKNOWLEDGEMENTS}

This research was performed under the auspices of the U.S. Department of Energy by the University of California, Lawrence Livermore National Laboratory under Contract W-7405-ENG-48, and also supported in part by the National Science Foundation Science and Technology Center for Adaptive Optics, managed by the University of California at Santa Cruz under cooperative agreement No. AST - 9876783. Support for this work was also provided by a grant from the Gordon and Betty Moore Foundation to the Regents of the University of California, Santa Cruz, on behalf of the Laboratory for Adaptive Optics. The content of the information does not necessarily reflect the position or the policy of the Gordon and Betty Moore Foundation, and no official endorsement should be inferred.

\section{References}

Sivaramakrishnan, A., J. P. Lloyd, P. E. Hodge et al., Astrophysical Journal Supplement Series 581 (1), L59 2002.

Macintosh, B., J. R. Graham, L. A. Poyneer et al., presented at the Technquies and Instrumentation for Detection of Exoplanets, 2003.

Macintosh, B., J. R. Graham, B. J. Bauman et al., presented at the Astronomical Telescopes and Instrumentation, 2004.

Jacquinot, P. and B. Roizen-Dossier, in Progress in Optics (North-Holland Publishing Company, Amsterdam, 1964), Vol. 3, pp. 31.

Kasdin, N. J., R. J. Vanderbei, D. N. Spergel et al., Astrophysical Journal 582 (2), 11472003.

Sivaramakrishnan, A., C. D. Koresko, R. B. Makidon et al., Astrophysical Journal 552 (1), 3972001.

Sommargren, G., in OSA Trends in Optics and Photonics: Extreme Ultraviolet Lithography, edited by G.D. Kubiak and D. R. Kania (Optical Soceity of America, Washington, DC, 1996), Vol. 4, pp. 108. Sommargren, G., D. Phillion, M. Johnson et al., presented at the Emerging Lithographic Technologies VI, 2002.

Poyneer, L. A. and B. Macintosh, Journal of the Optical Society of America A-Optics \& Image Science 21 (5), 8102004. 